\title{
Cinética de fermentação ruminal in vitro de diferentes co-produtos agroindustriais utilizados
}

\section{em dietas para ruminantes}

\author{
Ferro, M.M ${ }^{1}$; Costa, F.G. ${ }^{2}$; de Moura, D.C. ${ }^{1}$; Costa, R.V. ${ }^{3}$; de Oliveira, E.B. ${ }^{1}$ e Ferro, R.M. ${ }^{4}$
}

'Programa de Pós-Graduação em Ciência Animal. Universidade Federal de Mato Grosso. Cuiabá. MT. Brazil.

2Programa de Pós-Graduação em Zootecnia. Universidade Federal de Góias. Goiânia. GO. Brazil.

3Programa de Pós-Graduação em Zootecnia. Universidade Estadual de São Paulo. Jaboticabal. SP. Brazil.

${ }^{4}$ Médico Veterinário. Primavera do Leste. MT. Brazil.

\section{PALAVRAS CHAVE ADICIONAIS}

Degradação ruminal.

Farelo de gérmen de milho.

Farelo de girassol.

Grão seco da destilaria do milho.

\section{ADDITIONAL KEYWORDS}

Corn germ meal.

Dried distillers grain of corn.

Ruminal degradation.

Sunflower meal.

\section{RESUMO}

O objetivo deste trabalho foi avaliar o valor nutricional de três co-produtos agroindustriais em dietas para ovinos em terminação utilizando a técnica de produção de gases in vitro. Os tratamentos experimentais foram constituídos pela inclusão de três diferentes co-produtos nas dietas sendo eles grão seco da destilaria do milho, farelo de gérmen de milho e farelo de girassol em níveis de 0, 10, 20 e 30\%. A cinética da produção cumulativa dos gases foi analisada empregando-se o modelo logístico bicompartimental. Os parâmetros foram analisados através da análise de variância, e quando significativo, contrastes ortogonais foram utilizados para partição específica dos efeitos de níveis de inclusão dos alimentos as dietas. A inclusão do farelo de gérmen de milho nas dietas foi observado comportamento quadrático $(\mathrm{P}<0,05)$ para a produção de gases e a taxa de degradação da fração de rápida digestão, redução linear $(\mathrm{P}<0,05)$ para a produção de gases da fração de lenta digestão e nenhuma diferença significativa $(P>0,05)$ para a taxa de degradação desta mesma fração. A digestibilidade ruminal in vitro reduziu linearmente $(P<0,05)$ com a inclusão dos níveis deste co-produto. Quando se incluiu grão seco da destilaria do milho nas dietas a produção de gases da fração de rápida digestão apresentou redução linear $(\mathrm{P}<0,05)$, a taxa de degradação desta mesma fração não apresentov efeito significativo $(P>0,05)$, a produção de gases da fração de lenta digestão apresentou aumento linear $(P<0,05)$ e a taxa de degradação desta fração que não se alterou $(P>0,05)$. A digestibilidade ruminal in vitro da $M S$ não alterou $(P>0,05)$ com a inclusão deste co-produto. $A$ inclusão do farelo de girassol gerou reduziu linear $(P<0,05)$ na produção de gases da fração de rápida digestão, efeito não significativo $(P>0,05)$ para a taxa de degradação desta mesma fração, comportamento quadrático $(P<0,05)$ para a produção de gases da fração de lenta digestão e efeito quadrático $(\mathrm{P}<0,05)$ para a taxa de degradação desta fração. Não houve efeito significativo $(\mathrm{P}<0,05)$ para a digestibilidade ruminal da matéria seca in vitro. Conclui-se que embora a inclusão dos co-produtos nas dietas tenha alterado um ou mais parâmetros cinéticos de fermentação ruminal, a digestibilidade ruminal in vitro não se alterou significativamente.

\section{In vitro ruminal fermentation kinetics of different agroindustrial co-products used in ruminant} diets

\section{SUMMARY}

The main of this work was to evaluate the nutritional value of three agroindustrial co-products in finishing diets for sheep using the technique of in vitro gas production. The experimental treatments consisted of the inclusion of three different co-products in the diets: corn germ meal, corn germ meal and sunflower meal at levels of $0,10,20$ and $30 \%$. The kinetics of cumulative gas production was analyzed using the bicompartmental logistic model. The parameters were analyzed through analysis of variance, and when significant, orthogonal contrasts were used for specific partitioning of the effects of dietary inclusion levels on diets. The addition of maize germ meal in the diets, a quadratic behavior $(P<0.05)$ was observed for the gas production and the rate of degradation of the fast digestion fraction, linear reduction $(P<0.05)$ for the production of gas from the slow digestion fraction and no significant difference $(P>0.05)$ for the degradation rate of this same fraction. In vitro ruminal digestibility reduced linearly $(P<0.05)$ with inclusion of the levels of this co-product. When dry corn distillery was included in the diets, the production of gases of the fast digestion fraction showed a linear reduction $(P<0.05)$, the degradation rate of this same fraction did not show a significant effect $(P>0.05)(P<0.05)$ and the rate of degradation of this fraction did not change $(P>0.05)$. In vitro ruminal digestibility of $D M$ did not change $(P>0.05)$ with inclusion of this coproduct. The inclusion of sunflower meal resulted in a linear reduction $(P<0.05)$ in the gas production of the fast digestion fraction, a non significant effect $(P>0.05)$ for the degradation rate of this same fraction, $0.05)$ for the production of slow digestion fraction and quadratic effect $(P<0.05)$ for the degradation rate of this fraction. There was no significant effect $(P<0.05)$ for ruminal digestibility of dry matter in vitro. It was concluded that although the inclusion of the co-products in the diets altered one or more kinetic parameters of ruminal fermentation, ruminal digestibility in vitro did not change significantly.

\author{
Cronología del artículo. \\ Recibido/Received: 11.06 .2018 \\ Aceptado/Accepted: 23.02.2021 \\ On-line: 15.04 .2021 \\ Correspondencia a los autores/Contact e-mail: \\ mmf_zootecnia@yahoo.com.br
}




\section{INTRODUÇÃO}

A utilização de co-produtos agroindustriais de origem vegetal na alimentação de ruminantes apresenta como vantagens o aumento na oferta de ingredientes tradicionalmente utilizados em rações de não ruminantes e também alimentação humana, como milho e soja. Outro ponto é a possibilidade de negociação de preços com consequente otimização dos custos, já que não se apresentam como commodities e também colaboram com a redução da poluição ambiental, uma vez que, a forma de descarte desses alimentos ainda não está estabelecida.

Muitos co-produtos agroindustriais não apresentam informações suficientes quanto a sua composição química e níveis adequados de inclusão, levando a necessidade de se estudar a sua viabilidade de utilização e quantificar as respostas nutricionais e fisiológicas em animais ruminantes. A avaliação do consumo e digestibilidade dos nutrientes e a utilização dos nutrientes nos diferentes compartimentos do trato digestivo são de extrema importância para obtenção de um valor nutritivo dos alimentos. Um dos principais objetivos na nutrição de ruminantes é maximizar a produção de proteína microbiana através da sincronização da degradação de energia e proteína no rúmen.

$\mathrm{O}$ valor nutritivo de um alimento depende de diversos fatores, entre eles a sua composição química e o aproveitamento de seus nutrientes pelos animais, uma vez que, nos animais ruminantes esse aproveitamento é resultante da simbiose entre o animal e a microbiota ruminal (Santo et al., 2017). Desta forma, o conhecimento da dinâmica de degradação ruminal de diversos alimentos é fundamental para adequação das dietas quanto as informações relativas às proporções das frações dos alimentos, bem como sua taxa de digestão e perdas decorrentes da fermentação ruminal (Goes et al., 2010).

Dentre as alternativas, o beneficiamento de produtos agroindustriais tem produzido diversos resíduos com potencial de utilização na nutrição de animais ruminantes, tal como, farelo de girassol, farelo de gérmen de milho e grão seco da destilaria do milho. Neste contexto hipotetizamos que estes co-produtos ao serem inclusos nas dietas para ruminantes não afetam os parâmetros cinéticos de degradação e a digestibilidade in vitro da matéria seca. Assim objetivou-se investigar os parâmetros cinéticos de fermentação in vitro de três diferentes co-produtos agroindustriais inclusos em dietas para ruminantes.

\section{MATERIAL E MÉTODOS}

\section{LOCAL E ANIMAIS}

As incubações foram realizadas nas dependências do laboratório de Nutrição Animal da Universidade Federal de Mato Grosso - Campus Cuiabá-MT. Foi utilizado dois ovinos adultos, canulados no rúmen para coleta do líquido ruminal, mantidos no setor de Experimentação de ruminantes na cidade de Santo Antônio do Leverger, pertencente a Universidade Federal de Mato Grosso.
Dietas experimentais e análises quimico-bromatológicas

As dietas experimentais foram compostas por 30\% de volumoso (silagem de milho) e $70 \%$ de concentrado, na matéria seca, sendo as mesmas isoprotéicas com $15 \%$ de PB (NRC, 2007). Os tratamentos experimentais foram constituídos pela inclusão de três diferentes co-produtos nas dietas sendo eles grão seco da destilaria do milho, farelo de gérmen de milho e farelo de girassol em níveis de 0, 10, 20 e 30\%. A composição das dietas está descrita na Tabela I.

Todas as amostras foram analisadas de acordo com procedimentos de análise padrão do Instituto Nacional Brasileiro de Ciência e Tecnologia na Ciência Animal (INCT-CA) (Detmann et al., 2012). As amostras dos alimentos volumosos foram pré-secas em estufa de ventilação forçada sob $55^{\circ} \mathrm{C}$ durante 72 horas para determinação da amostra seca ao ar (INCT-CA G-001/1). Em seguida todos os alimentos foram submetidos a estufa $105^{\circ} \mathrm{C}$ para determinação da matéria seca definitiva (INCT-CA G-003/1). Posteriormente, todos os alimentos foram moídos em peneira de $1 \mathrm{~mm}$ para serem analisados para proteína bruta (INCT-CA N-001/1), matéria mineral (INCT-CA M-001/1), extrato etéreo (INCT-CA G-004/1), fibra detergente neutro (INCT-CA F-002/1). Os carboidratos totais (CT) foram calculados de acordo com Sniffen et al. (1992). A composição química-bromatológica dos ingredientes que compuseram as dietas experimentais estão apresentados na Tabela II.

\section{ENSAIOS IN VITRO}

Para desenvolvimento das incubações, os alimentos foram moídos a $1 \mathrm{~mm}$ conforme (Pell e Schofield, 1993), em seguida foram pesados $500 \mathrm{mg}$ de amostra seca ao ar (ASA), que foram alocadas em frascos de vidro cor âmbar com capacidade de $120 \mathrm{~mL}$.

\begin{tabular}{|c|c|c|c|c|}
\hline \multirow[t]{2}{*}{ Ingrediente $\left(\mathrm{kg}^{-1}\right)$} & \multicolumn{4}{|c|}{ Níveis de inclusão $\left(\mathrm{g} \mathrm{kg}^{-1}\right)$} \\
\hline & 0 & 100 & 200 & 300 \\
\hline \multicolumn{5}{|l|}{ Farelo de Gérmen de milho } \\
\hline Silagem de milho & 0,300 & 0,300 & 0,300 & 0,300 \\
\hline Farelo de soja & 0,200 & 0,190 & 0,180 & 0,160 \\
\hline Milho & 0,500 & 0,400 & 0,310 & 0,220 \\
\hline Farelo de Gérmen de milho & 0,000 & 0,110 & 0,210 & 0,320 \\
\hline \multicolumn{5}{|l|}{ Grão seco da destilaria do milho } \\
\hline Silagem de milho & 0,300 & 0,300 & 0,300 & 0,300 \\
\hline Farelo de soja & 0,200 & 0,130 & 0,070 & 0,000 \\
\hline Milho & 0,500 & 0,460 & 0,420 & 0,380 \\
\hline Grão seco destilaria do milho & 0,000 & 0,110 & 0,210 & 0,320 \\
\hline \multicolumn{5}{|l|}{ Farelo de girassol } \\
\hline Silagem de milho & 0,300 & 0,300 & 0,300 & 0,300 \\
\hline Farelo de soja & 0,200 & 0,130 & 0,070 & 0,000 \\
\hline Milho & 0,500 & 0,460 & 0,420 & 0,380 \\
\hline Farelo de girassol & 0,000 & 0,110 & 0,210 & 0,320 \\
\hline
\end{tabular}


O meio utilizado foi composto seguindo recomendação de McDougall (1949), preparado para manter o pH entre 6,9 a 7,0, com aspersão constante de $\mathrm{CO}_{2}$. Imediatamente após a adição do meio, os frascos receberam tampa de borracha e lacre de alumínio.

O inóculo ruminal foi coletado 2 horas após a alimentação da manhã, a partir da mistura homogênea do conteúdo ruminal de diferentes regiões do rúmen. Após a coleta da amostra composta de conteúdo sólido ruminal, a fase líquida foi removida da sólida por sacos de tecido, acondicionando em garrafa térmica pré-aquecida com fechamento hermético e transportado imediatamente para o laboratório sendo toda a sua manipulação realizada sob aspersão de $\mathrm{CO}_{2}$ (livre de oxigênio). Posteriormente o material foi filtrado em quatro camadas de gaze e mantido a $39^{\circ} \mathrm{C}$ por $10 \mathrm{mi}-$ nutos em repouso em frasco transparente com infusão contínua de $\mathrm{CO}_{2}$.

Aos frascos foram adicionados $40 \mathrm{~mL}$ de solução meio, onde meia hora após, foram adicionados $10 \mathrm{~mL}$ de inoculo ruminal sob aspersão de $\mathrm{CO}_{2}$ utilizando seringa e agulha hipodérmica ( 40 x 12 mm; (18G x 1 1 1/2") e mantidos à temperatura de $39^{\circ} \mathrm{C}$ em banho maria com agitação orbital constante (Malafaia, 1997).

\section{Produção tOtAL de GASES}

A produção total de gases foi mensurada utilizando sistema semi-automático (Theodorou et al., 1994), por meio de transdutor de pressão (psi - pressão por polegada quadrada) (Modelo DPI 705 Series Digital; Marca GE, Leicester, Inglaterra) para aferir a pressão absoluta em mega pascal (MPa) nos seguintes tempos: 1, 2, 3, 4, 5, 6, 8, 10, 12, 24, 36, 48, 60, 72 e 96 horas. Para descontar o volume de gases oriundo do líquido de rúmen e da solução tampão, dois frascos foram incubados sem amostra (branco). Dessa forma, para cada tempo de leitura, o volume de gases dos frascos com amostra foi subtraído do volume dos frascos sem amostra.

Com o somatório do volume de gases para cada tempo de leitura, foram estabelecidas as curvas de produção cumulativa dos gases. A conversão de psi para $\mathrm{mL}$ foi feita a partir da equação de regressão linear $(Y=a \pm b x)$ em que o coeficiente " $b$ " da equação possibilitou a correção e transformação de pressão (psi) em volume de gases $(\mathrm{mL})$ corrigido para a pressão barométrica do dia. Para isso, injetou- se volume conhecido de gases em frascos mantidos sob as mesmas condições das amostras incubadas. Conforme a relação da amostra utilizada gerando uma curva conforme a pressão correspondente ao volume injetado $(50 \mathrm{~mL})$, sendo as mesmas medidas e utilizadas para a obtenção da equação de regressão entre pressão e volume de gases.

A cinética da produção cumulativa dos gases foi analisada empregando-se o modelo logístico bicompartimental onde: $\mathrm{V}(\mathrm{t})=\mathrm{Vf}_{1} /\left(1+\exp \left(2-4^{*} \mathrm{c}_{1}{ }^{*}(\mathrm{~T}-\mathrm{L})\right)\right)$ $+\mathrm{Vf}_{2} /\left(1+\exp \left(2-4^{*} \mathrm{c}_{2}{ }^{*}(\mathrm{~T}-\mathrm{L})\right)\right)$, no qual $\mathrm{V}(\mathrm{t})$ é o volume de gases acumulado no tempo $\mathrm{t} ; \mathrm{Vf}_{1}$, o volume de gases oriundo da fração de rápida digestão; $\mathrm{c}_{1}\left(\mathrm{~h}^{-1}\right)$, a taxa de degradação da fração de rápida digestão; $\mathrm{T}$, o tempo (h); L, a latência; $\mathrm{Vf}_{2}$, o volume de gases da fração de lenta digestão; $\mathrm{c}_{2}\left(\mathrm{~h}^{-1}\right)$, a taxa de degradação da fração de lenta digestão, ambos descrito por Schofield et al. (1994)measured by computer-interfaced pressure sensors, was used to follow the digestion of a crystalline processed cellulose, a bacterial cellulose, and mixtures of these substrates by mixed ruminal bacteria. A first-order, substrate limited model (simple exponential with lag.

\section{ESTIMATIVA DE DIGESTIBILIDADE IN VITRO}

Para estimativa da digestibilidade da MS das dietas experimentais e dos alimentos, ao termino de cada incubação o resíduo da digestão in vitro foi filtrado utilizando-se cadinhos filtrantes de porosidade $3 \mathrm{~mm}$ e em seguida colocados em estufa à $105^{\circ} \mathrm{C}$ para determinação da MS (método No 934.01) (AOAC, 1990). A digestibilidade da MS foi calculada dividindo-se o resíduo da incubação pelo total de amostra incubada.

\section{ANÁlISE ESTATÍSTICA}

Para cada co-produto avaliado foram conduzidas três incubações com 2 repetições por tratamento, totalizando 6 frascos por tratamento. A incubação foi utilizada como bloco, e as repetições dentro de cada bloco foram efetuadas médias.

Os parâmetros foram analisados através da análise de variância, e quando significativo, contrastes ortogonais foram utilizados para partição específica dos efeitos de níveis de inclusão dos alimentos as dietas experimentais em linear, quadrático e cúbico. Em todas as análises, significâncias foram declaradas a $\mathrm{P}<0,05$, utilizando o Proc Mixed do SAS versão 9.2.

Tabela II. Composição química-bromatológica dos ingredientes das dietas experimentais (Chemical-bromatological composition of the ingredients of experimental diets).

\begin{tabular}{|c|c|c|c|c|c|c|}
\hline \multirow{2}{*}{ Componentes } & \multicolumn{6}{|c|}{ Alimentos $^{1}\left(\mathrm{~g} \mathrm{~kg}^{-1} \mathrm{MS}\right)$} \\
\hline & SM & FS & MI & DDGM & FGM & $\mathrm{FG}$ \\
\hline Matéria seca & 0,300 & 0,910 & 0,876 & 0,917 & 0,906 & 0,917 \\
\hline Proteína bruta & 0,092 & 0,541 & 0,085 & 0,360 & 0,111 & 0,391 \\
\hline Extrato etéreo & 0,020 & 0,021 & 0,052 & 0,076 & 0,050 & 0,020 \\
\hline Fibra Detergente Neutro & 0,552 & 0,110 & 0,088 & 0,131 & 0,254 & 0,653 \\
\hline Carboidratos não fibrosos & 0,317 & 0,149 & 0,634 & 0,097 & 0,458 & 0,208 \\
\hline
\end{tabular}

1SM: silagem de milho; FS: farelo de soja; MI: milho moído; DDGM: grão seco da destilaria do milho; FG: farelo de girassol; FGM: farelo de gérmen de milho 


\section{RESULTADOS E DISCUSSÃO}

O milho grão apresentou produção total de gases superior aos outros alimentos durante todos os tempos de incubação.Dentre os co-produtos avaliados, o farelo de gérmen de milho apresentou uma produção total de gases alta nas primeiras 36 horas de incubação, entretanto, nas horas seguintes o farelo de soja ultrapassou essa produção de gases. $\mathrm{O}$ farelo de girassol sempre se manteve abaixo de todos os alimentos avaliados, e o grão seco da destilaria do milho manteve-se de forma intermediária (Figura 1).

Dentre os co-produtos avaliados o farelo de gérmen de milho foi o que apresentou maior produção de gases da fração de rápida degradação $(38,57 \mathrm{~mL})$, seguido do farelo de girassol $(21,66 \mathrm{~mL})$ e o grão seco da destilaria do milho $(8,68 \mathrm{~mL})$. Uma maior taxa de degradação da fração de rápida digestão foi obtida para o farelo de gérmen de milho que apresentou uma taxa de 15,88\% da matéria seca sendo degradada hora-1.

Para a fração de lenta degradação, o milho grão apresentou maior produção de gases com uma produção média de 129,20 mL. Para esta mesma fração a maior taxa de degradação foi observada para o milho grão, e dentre os co-produtos avaliados, o farelo de gérmen de milho foi o que apresentou maior taxa de degradação com $2,77 \%$ da matéria seca sendo degradada hora-1 ${ }^{-1}$ Tabela III).

Fortaleza et al., (2009) avaliando diferentes alimentos concentrados obtiveram uma taxa de degradação total da MS de 5,74 e 5,71\% para farelo de soja e milho, respectivamente. Valores inferiores foram obtidos por Pegoraro et al., (2017) para farelo de soja (2,64\%), farelo de algodão (2,37\%), torta de girassol (2,65\%) e DDG do milho (3,51\%) e Teixeira (2014) que obtiveram taxas de $2,67 \%$ para DDG do milho e 6,65\% para farelo de soja.

A menor latência foi observada para farelo de girassol com 1,84 horas e uma maior latência foi observada para o grão seco da destilaria do milho com 13,48 horas.

A digestibilidade ruminal in vitro da matéria seca foi maior para milho grão com 95,33\%, e dentre os coprodutos avaliados, o grão seco da destilaria do milho

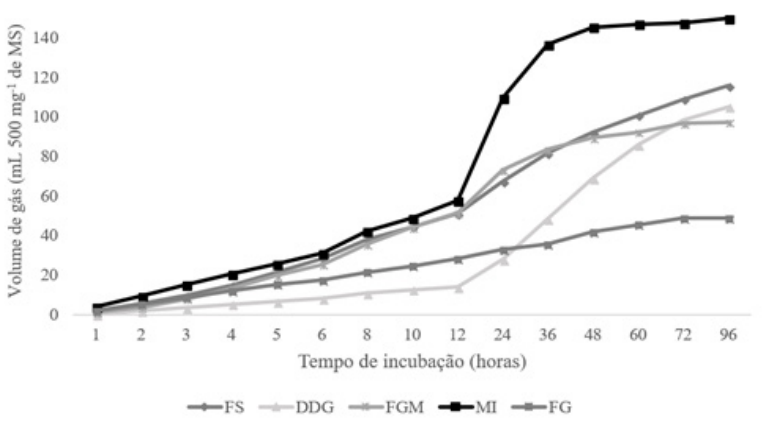

Figura 1. Produçao cumulativa de gás dos alimentos farelo de soja (FS), grão seco da destilaría do milho (DDG), farelo de gérmen de milho (FMG), milho grão (MI) e farelo de girasol (FG) (Accumulated gas production from food soybean meal (FS), corn distillery dry grain (DDG), corn germ meal (FMG), corn grain (MI) and sunflower flour (FG). foi o que apresentou maior digestibilidade ruminal com 63,95\% (Tabela III). Este valor de digestibilidade do milho também foi encontrado por Fortaleza et al., (2009) que obtiverem um degradação aparente média de $99,15 \%$ para o milho triturado.

\section{FARELO DE GÉRMEN DE MILHO}

A Figura 2 mostra que os níveis de inclusão de farelo de gérmen de milho nas primeiras doze horas de fermentação foram bastante similares. Entretanto, a inclusão de $0 \%$ de farelo de gérmen de milho aumentou considerável a produção de gases após as 12 horas de fermentação, o que pode ser explicado pela alta inclusão de milho neste nível, uma vez que, dentre os alimentos que formaram as dietas, o milho grão é o que vem apresentando maior produção de gases.

A produção de gases e a taxa de degradação da fração de rápida digestão apresentaram comportamento quadrático $(\mathrm{P}<0,05)$ a medida que incluiu o farelo de gérmen de milho nas dietas. A produção de gases da fração de lenta digestão apresentou redução linear $(\mathrm{P}<0,05)$, enquanto, a taxa de degradação desta mesma fração não apresentou diferença significativa $(\mathrm{P}>0,05)$ com os níveis de inclusão do farelo de gérmen de milho. A latência reduziu linearmente $(\mathrm{P}<0,05)$ com a inclusão do farelo de gérmen de milho.

A redução linear $(\mathrm{P}<0,05)$ na digestibilidade ruminal in vitro com a inclusão dos níveis deste co-produto ja era esperada devido a sua reduzida taxa de degradação comparativamnete ao milho e farelo de soja que foram os alimentos substituídos a medida que foi incluindo o co-produto (Tabela IV).

\section{GRÃO SECO DA DESTILARIA DO MILHO}

Tabela III. Parâmetros cinéticos de fermentação ruminal in vitro e digestibilidade ruminal da MS pela técnica cumulativa de gases dos alimentos (Kinetic parameters of ruminal fermentation in vitro and ruminal digestibility of DM by cumulative food gas technique).

\begin{tabular}{lcccccc}
\hline $\begin{array}{l}\text { Parâme- } \\
\text { tros }^{1}\end{array}$ & SM & FS & MI & DDGM & FGM & FG \\
VGCNF & 39,14 & 38,70 & 18,55 & 8,68 & 38,57 & 21,66 \\
KdCNF & 0,0943 & 0,1671 & 0,3138 & 0,0628 & 0,1588 & 0,1507 \\
VGCHOF & 77,50 & 75,40 & 129,20 & 98,57 & 56,24 & 27,99 \\
KdCHOF & 0,0134 & 0,0169 & 0,0355 & 0,0180 & 0,0277 & 0,0160 \\
Latência & 4,34 & 3,66 & 3,90 & 13,48 & 3,60 & 1,84 \\
VGtotal & 116,64 & 114,10 & 147,75 & 107,25 & 94,81 & 49,65 \\
Digestibilidade ruminal in vitro da MS & & & \\
DIVMS & 67,42 & 90,66 & 95,33 & 63,95 & 60,95 & 59,21 \\
\hline
\end{tabular}

VFCNF (ml $\left.500 \mathrm{mg}^{-1} \mathrm{MS}\right)$ : volume final de gás referente à fração de rápida digestão (CNF); $\mathrm{KdCNF}\left(\% \mathrm{~h}^{-1}\right)$ : taxa de degradação referente à fração de rápida digestão (CNF); VFCHOF (ml $\left.500 \mathrm{mg}^{-1} \mathrm{MS}\right)$ : volume final de gás referente à fração de lenta digestão (CHOF); $\mathrm{KdCHOF}\left(\% \mathrm{~h}^{-1}\right)$ : taxa de degradação referente à fração de lenta degradação (CHOF); Latência: horas; DIVMS: digestibilidade in vitro de matéria seca após 96 horas de incubação; ${ }^{2} \mathrm{FS}$ : Farelo de soja; DDGM: Grão seco da destilaria do milho; FGM: Farelo de gérmen de milho; MI: Milho grão; FG: Farelo de girassol 


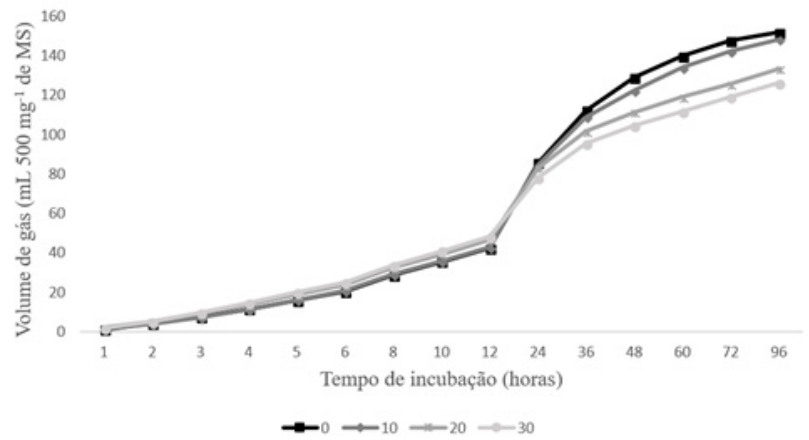

Figura 2. Produçao cumulativa de gases para as dietas experimentais com incluso do farelo de gérmen de milho (Accumulated gas production for experimental diets including maize germ meal).

Diferentemente do observado para o farelo de gérmen de milho, a inclusão do grão seco de destilaria levou a uma menor produção de gases desde o início da incubação, e somente a partir de 60 horas após a fermentação os tratamentos começaram a se equiparar (Figura 3), mostrando que o DDG de milho apresenta lenta e incompleta digestão no rúmen.

A produção de gases da fração de rápida digestão apresentou redução linear $(\mathrm{P}<0,05)$ com a inclusão dos níveis de grão de destilaria do milho. Entretanto, essa menor produção de gases não levou a alteração da taxa de degradação desta mesma fração, que não apresentou efeito significativo $(\mathrm{P}>0,05)$ (Tabela V). Essa redução na produção de gases pode estar ligada a não utilização do farelo de soja na dieta com $30 \%$ de DDG do milho devido o mesmo ter sido totalmente substituído pelo co-produto.

A produção de gases da fração de lenta digestão apresentou aumento linear $(\mathrm{P}<0,05)$, porém, não o suficiente para alterar a taxa de degradação desta fração que não se alterou $(\mathrm{P}>0,05)$ com o aumento da inclusão deste co-produto. A latência aumentou linearmente $(\mathrm{P}<0,05)$ com o a inclusão do grão seco da destilaria do milho.

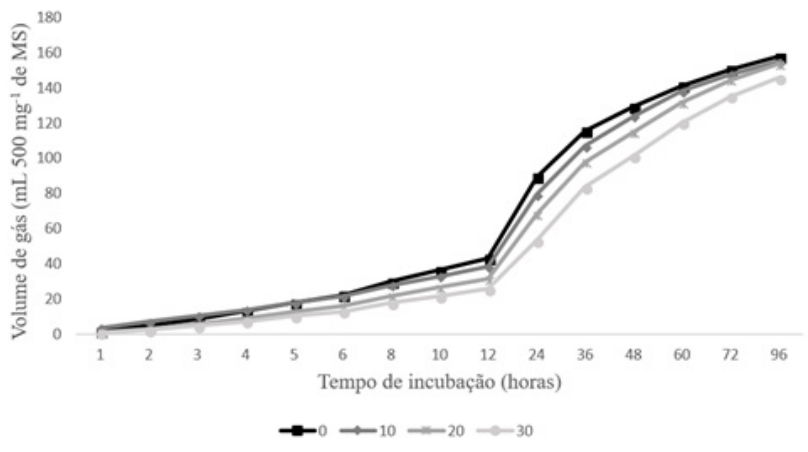

Figura 3. Producão cumulativa de gases as dietas experimentais com incluso do grão seco da destilaría do milho (Accumulated gas production in experimental diets including dry grain from the corn distillery).

Para a digestibilidade ruminal in vitro da MS não houve diferença significativa $(\mathrm{P}>0,05)$ para as dietas testadas, resposta esta contrária ao esperado, uma vez que, esperava-se redução na digestibilidade a medida que incluia-se este co-produto.

\section{FARELO DE GIRASSOL}

As produções de gases foram similares com a inclusão do farelo de girassol nas primeiras 24 horas de incubação, entretanto, o nível $0 \%$ de inclusão mantevese acima dos outros níveis de inclusão nas horas seguintes de fermentação (Figura 4).

A produção de gases da fração de rápida digestão reduziu linearmente $(P<0,05)$ a medida que incluiu farelo de girassol nas dietas, porém, esta redução não alterou $(P>0,05)$ a taxa de degradação desta mesma fração. A produção de gases da fração de lenta digestão apresentou comportamento quadrático $(\mathrm{P}<0,05)$ com a inclusão dos níveis de farelo de girassol, levando a um mesmo efeito quadrático $(\mathrm{P}<0,05)$ para a taxa de degradação desta fração. Não houve efeito $(\mathrm{P}<0,05)$ na latência e na digestibilidade ruminal da matéria seca in vitro (Tabela VI).

Dietas similares usando torta de girassol foram avaliadas por Silva et al., (2015) onde observaram que

Tabela IV. Parâmetros cinéticos de fermentação ruminal in vitro pela técnica cumulativa de gases das dietas contendo níveis de inclusão do farelo de gérmen de milho (Kinetic parameters of ruminal fermentation in vitro by the cumulative technique of diet gases containing inclusion levels of corn germ meal).

\begin{tabular}{|c|c|c|c|c|c|c|c|c|}
\hline \multirow[t]{2}{*}{ Parâmetros } & \multicolumn{4}{|c|}{ Níveis de inclusão $\left(\mathrm{g} \mathrm{kg}^{-1}\right)$} & \multirow[t]{2}{*}{ EPM } & \multicolumn{3}{|l|}{ Valor-P } \\
\hline & 0 & 100 & 200 & 300 & & Linear & Quadrático & Cúbico \\
\hline VGCNF & 81,62 & 88,92 & 85,93 & 71,56 & 5,23 & 0,0631 & 0,0076 & 0,9496 \\
\hline KdCNF & 0,0657 & 0,0542 & 0,0593 & 0,0795 & 0,0062 & 0,1037 & 0,0047 & 0,8038 \\
\hline VGCHOF & 89,89 & 83,90 & 70,44 & 76,70 & 6,84 & 0,0404 & 0,2741 & 0,2717 \\
\hline $\mathrm{KdCHOF}$ & 0,0179 & 0,0150 & 0,0165 & 0,0176 & 0,0013 & 0,9063 & 0,0537 & 0,2798 \\
\hline Latência & 6,95 & 6,88 & 5,69 & 4,82 & 0,6002 & 0,0009 & 0,3973 & 0,4931 \\
\hline \multicolumn{9}{|c|}{ Digestibilidade ruminal in vitro da MS (\%) } \\
\hline DIVMS & 89,41 & 83,56 & 80,53 & 75,34 & 4,37 & 0,0005 & 0,3421 & 0,4206 \\
\hline \multicolumn{9}{|c|}{$\begin{array}{l}\left.\text { VFCNF (ml } 500 \mathrm{mg}^{-1} \mathrm{MS}\right) \text { : volume final de gás referente à fração de rápida digestão (CNF); KdCNF }\left(\% \mathrm{~h}^{-1}\right) \text { : taxa de degradação referente } \\
\left.\text { à fração de rápida digestão (CNF); VFCHOF (ml } 500 \mathrm{mg}^{-1} \mathrm{MS}\right) \text { : volume final de gás referente à fração de lenta digestão (CHOF); KdCHOF } \\
\left(\% \mathrm{~h}^{-1}\right) \text { : taxa de degradação referente à fração de lenta degradação (CHOF); Latência: horas; DIVMS: digestibilidade in vitro de matéria } \\
\text { seca após } 96 \text { horas de incubação; DIVMS: digestibilidade in vitro da MS após } 96 \text { horas de incubação }\end{array}$} \\
\hline
\end{tabular}




\begin{tabular}{|c|c|c|c|c|c|c|c|c|}
\hline \multirow[t]{2}{*}{ Parâmetros } & \multicolumn{4}{|c|}{ Níveis de inclusão $\left(\mathrm{g} \mathrm{kg}^{-1}\right)$} & \multirow{2}{*}{ EPM } & \multicolumn{3}{|c|}{ Valor-P } \\
\hline & 0 & 100 & 200 & 300 & & Linear & Quadrático & Cúbico \\
\hline VGCNF & 99,91 & 81,65 & 82,55 & 72,14 & 8,92 & 0,0023 & 0,4636 & 0,2101 \\
\hline KdCNF & 0,0369 & 0,0394 & 0,0381 & 0,0367 & 0,0069 & 0,9127 & 0,6339 & 0,8426 \\
\hline VGCHOF & 109,63 & 123,89 & 122,67 & 126,31 & 8,21 & 0,0413 & 0,2901 & 0,3529 \\
\hline $\mathrm{KdCHOF}$ & 0,0174 & 0,0171 & 0,0166 & 0,0164 & 0,0006 & 0,0794 & 0,9812 & 0,8903 \\
\hline Latência & 12,21 & 12,42 & 14,93 & 17,01 & 1,24 & $<0,0001$ & 0,2125 & 0,4099 \\
\hline \multicolumn{9}{|c|}{ Digestibilidade ruminal in vitro da MS } \\
\hline DIVMS & 74,31 & 68,32 & 68,33 & 63,09 & 7,89 & 0,1291 & 0,9362 & 0,5943 \\
\hline \multicolumn{9}{|c|}{$\begin{array}{l}\left.\text { VFCNF (ml } 500 \mathrm{mg}^{-1} \mathrm{MS}\right) \text { : volume final de gás referente à fração de rápida digestão (CNF); KdCNF }\left(\% \mathrm{~h}^{-1}\right) \text { : taxa de degradação referente } \\
\left.\text { à fração de rápida digestão (CNF); VFCHOF (ml } 500 \mathrm{mg}^{-1} \mathrm{MS}\right) \text { : volume final de gás referente à fração de lenta digestão (CHOF); KdCHOF } \\
\left(\% \mathrm{~h}^{-1}\right) \text { t taxa de degradação referente à fração de lenta degradação (CHOF); Latência: horas; DIVMS: digestibilidade in vitro de matéria } \\
\text { seca após } 96 \text { horas de incubação; DIVMS: digestibilidade in vitro da MS após } 96 \text { horas de incubação }\end{array}$} \\
\hline
\end{tabular}

Tabela VI. Parâmetros cinéticos de fermentação ruminal in vitro pela técnica cumulativa de gases das dietas contendo níveis de inclusão do farelo de girassol (Kinetic parameters of ruminal fermentation in vitro by the cumulative technique of diet gases containing sunflower bran inclusion levels).

\begin{tabular}{|c|c|c|c|c|c|c|c|c|}
\hline \multirow[t]{2}{*}{ Parâmetros } & \multicolumn{4}{|c|}{ Níveis de inclusão $\left(\mathrm{g} \mathrm{kg}^{-1}\right)$} & \multirow{2}{*}{ EPM } & \multicolumn{3}{|c|}{ Valor-P } \\
\hline & 0 & 100 & 200 & 300 & & Linear & Quadrático & Cúbico \\
\hline VGCNF & 103,40 & 111,89 & 101,63 & 92,69 & 7,64 & 0,0481 & 0,0657 & 0,3152 \\
\hline KdCNF & 0,0610 & 0,0496 & 0,0557 & 0,0636 & 0,0088 & 0,5537 & 0,0854 & 0,5020 \\
\hline VGCHOF & 44,01 & 30,80 & 29,07 & 44,76 & 8,97 & 0,9826 & 0,0154 & 0,7975 \\
\hline $\mathrm{KdCHOF}$ & 0,0114 & 0,0087 & 0,0101 & 0,0123 & 0,0019 & 0,4319 & 0,0491 & 0,5211 \\
\hline Latência & 1,36 & 0,19 & 0,36 & 0,14 & 0,6361 & 0,0514 & 0,2105 & 0,2949 \\
\hline \multicolumn{9}{|c|}{ Digestibilidade ruminal in vitro da MS } \\
\hline DIVMS & 79,61 & 77,22 & 78,71 & 73,74 & 4,28 & 0,3033 & 0,5627 & 0,4542 \\
\hline
\end{tabular}

limitando o consumo de MS e consequentemente prejudicando o desempenho animal.

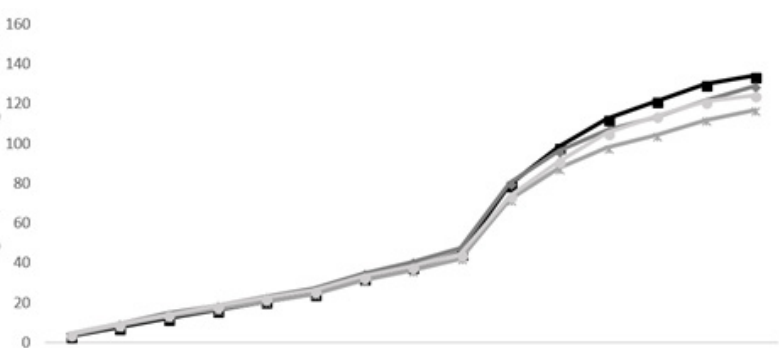

Figura 4. Produçao cumulativa de gases para as dietas experimentais com incluso do farelo de girasol (Accumulated gas production for experimental diets, including sunflower flour).

a inclusão de até 30\% não influenciou a produção de gases e nem a taxa de degradação da fração de rápida digestão, entretanto, reduziu a produção de gases e a taxa de degração de fração de lenta digestão. Essas caracteristícas foram discutidas por Mizubuti et al., (2011) que enfatizou que essa redução na taxa de degradação pode gerar efeito na repleção ruminal,

\section{CONCLUSÃO}

A utilização dos co-produtos nas dietas levou a alteração dos parâmetros cinéticos de fermentação ruminal in vitro. Entretanto, a digestibilidade in vitro da MS foi afetada somente quando o farelo de gérmen de milho foi incluso nas dietas, pois a inclusão do DDG de milho e o farelo de girassol não afetaram essa variável.

\section{BIBLIOGRAFIA}

AOAC. 1990. (Association of Official Analytical Chemists). "Official methods of analysis". 15.ed. Washington: AOAC.

Detmann, E, Souza, MA, Valadares Filho, SC, Queiroz, AC, Berchielli, Tा, Saliba, EOS, Cabral, LS, Pina, DS, Ladeira, MM, Azevedo, JAG. 2012 (Eds.) "Métodos para análise de alimentos". Visconde do Rio Branco: Suprema, 214p.

Fortaleza, APS, Silva, LDF, Ribeiro, ELA, Barbero, RP, Massaro Junior, FL, Santos, AX, Castro, VS, Castro, FAB 2009. "Degradabilidade ruminal In Situ dos componentes nutritivos de alguns suplementos concentrados 
usados na alimentação de bovinos", Semina: Ciências Agrárias, vol. 30, no. 2, pp. 481-496.

Goes, RHTB, Souza, KA, Patussi, RA, Cornelio, TC, Oliveira, ER, Brabes, KCS 2010 "Degradabilidade in situ dos grãos de crambe, girassol e soja, e de seus coprodutos em ovinos", Acta Scientiarum, vol. 32, no. 3, pp. 271-277.

McDougall, E. I. 1949. "Studies on Ruminant Saliva. 1. The Composition and Output of Sheep's Saliva", Biochemical Journal, vol. 43, no. 1, pp. 99-109.

Mizubuti, IY, Ribeiro, ELA, Pereira, ES, Pinto, AP, Franco, ALC, Syperreck, MA, Dórea, JRR, Cunha, GE, Capelari, MGM, Muniz, EB 2011 "Cinética de fermentação ruminal in vitro de alguns co-produtos gerados na cadeia produtiva do biodiesel pela técnica de produção de gás", Semina: Ciências Agrárias, vol. 32, no. 2, pp. 2021-2028.

Pegoraro, M, Silva, LDF, Fernandes Junior, F, Fortaleza, APS, Grandis, FA, Ribeiro, ELA, Castro, FAB 2017 "Avaliação nutricional e cinética de degradação in vitro de concentrados proteicos utilizados na alimentação de ruminantes", Revista Brasileira de Ciências Veterinárias, vol. 24, no. 1, pp. 31-38.

Pell, AN, Schofield, P 1993 "Computerized Monitoring of Gas Production to Measure Forage Digestion In Vitro", Journal Dairy Science, vol. 76, pp. 1063-1073.
Santo, AX, Silva, LDF, Lançanova, JAC, Ribeiro, ELA, Mizubuti, IY, Fortaleza, APS, Henz, ÉL, Junior, FLM 2017 "Fracionamento de carboidratos e proteínas, cinética de degradação ruminal in vitro pela técnica de produção de gás, de rações suplementares contendo torta de girassol", Arquivo Brasileiro de Medicina Veterinaria e Zootecnia, vol. 69, no.1, pp. 234-242.

Schofield, P, Pitt, RE, Pell, AN 1994 "Kinetics of fiber digestion from in vitro gas production", Journal of Animal Science, vol. 72, no.11, pp. 2980-2991

Silva, LHX, Goes, RHTB, Carneiro, MMY, Burin, PC, Oliveira, ER, Souza, KA, Ítavo, LCV, Branco, AF, Oliveiro, RT 2015 "Produção total de gases e degradabilidade in vitro de dietas com torta de girassol", Archivos de Zootecnia, vol. 64, no. 248, pp. 365-371.

Teixeira, UHG 2014 "Modelos Matemáticos para Estimação dos Parâmetros da Cinética de Degradação Ruminal de Coprodutos Protéicos", Dissertação (Mestrado em Ciência Animal) Universidade Federal do Mato Grosso, p. 42.

Theodorou, MK, Willians, BA, Dhanoa, MS, McAllan, AB, France, J 1994 "A simple gas production method using a pressure transducer to determine the fermentation kinetics of ruminant feeds", Animal Feed Science and Technology, vol. 48, no.3, pp. 185-197. 